\title{
Clinical course and prognosis of never-smokers with severe alpha-1-antitrypsin deficiency (PiZZ)
}

\author{
H A Tanash, ${ }^{1}$ P M Nilsson, ${ }^{2}$ J-Å Nilsson, ${ }^{1}$ E Piitulainen ${ }^{1}$
}

${ }^{1}$ Department of Respiratory Medicine, Malmö University Hospital, Lund University, Sweden; ${ }^{2}$ Department of Clinical Sciences, Malmö University Hospital, Lund University, Sweden

Correspondence to: Dr H Tanash, Department of Respiratory Medicine, Malmö University Hospital, Entrance 35, S-205 02, Malmö, Sweden; hanan.tanash@med.lu.se

Received 12 January 2008 Accepted 15 June 2008 Published Online First 5 August 2008

\begin{abstract}
Background: Previous studies of non-smoking individuals with severe $\alpha_{1}$-antitrypsin deficiency (PiZZ) have been sparse and included only a limited number of individuals, mostly identified by respiratory symptoms. The aim of this study was to estimate the prognosis of non-smoking PiZZ individuals and to analyse the most common causes of death by including a large number of individuals who had been identified by other means than respiratory symptoms.
\end{abstract}

Methods: The study included 568 non-smoking PiZZ subjects who were selected from the Swedish National AAT Deficiency Registry and followed up from 1991 to September 2007. Of these, 156 (27\%) were identified by respiratory symptoms (respiratory cases) and 412 were identified by extrapulmonary symptoms or screening (nonrespiratory cases).

Results: 93 subjects (16\%) died during the follow-up period. The specific standardised mortality rate (SMR) for the whole study population was $2.32(95 \% \mathrm{Cl} 1.87$ to 2.83) with no significant difference between men and women. The SMR was 2.55 (95\% Cl 1.91 to 2.83) for the respiratory cases and 2.07 (95\% Cl 1.49 to 2.81) for the non-respiratory cases. Further calculation of SMR for subgroups in the non-respiratory cases showed that the SMR was $0.70(95 \% \mathrm{Cl} 0.14$ to 2.04$)$ for individuals identified by family/population screening. Emphysema and liver cirrhosis were the most common causes of death (45\% and $28 \%$, respectively). Malignant transformation was found in $38 \%$ of the cases with cirrhosis.

Conclusion: Non-smoking PiZZ individuals identified by screening do not have an increased mortality risk compared with the Swedish general population.

Severe $\alpha_{1}$-antitrypsin (AAT) deficiency (PiZZ) is a relatively rare genetic disorder characterised by reduced serum levels of AAT. This genetic defect results in polymerisation of the AAT molecule in the hepatocytes, leading to accumulation of the Zprotein within the hepatocytes and decreased release into the circulation. In individuals with normal AAT genes, circulating AAT is distributed to the lungs to serve a protective function against the destruction of elastin, a protein that supports the lung structure. ${ }^{1-3}$

Panacinar emphysema is the most prevalent clinical disorder associated with severe AAT deficiency and the most frequent cause of disability and death..$^{46}$ Tobacco smoking is the major identifiable environmental risk factor for developing emphysema, which begins much earlier than usual emphysema that occurs in AAT-replete individuals. Liver disease is the second most frequent clinical manifestation of severe AAT deficiency and typically presents as cholestasis in infancy. ${ }^{78}$ In general, cirrhosis and carcinoma of the liver affect AATdeficient adults over the age of 50 years..$^{9-11}$

The natural history of never-smoking PiZZ individuals has been poorly documented. Previous studies have shown that PiZZ never-smokers have a remarkably delayed onset of symptoms and may have an almost normal life span. ${ }^{12-14}$ These studies have been sparse and included only a limited number of never-smokers.

The aim of this study was to estimate the prognosis of non-smoking PiZZ individuals and to analyse the most common causes of death by including a large number of individuals who had been identified for reasons other than respiratory symptoms.

\section{METHODS}

\section{Study population}

The Swedish National Registry of individuals with severe AAT deficiency was initiated in $1991 .{ }^{12}$ The main objective of the registry is to collect information on the natural history of AAT deficiency and to facilitate epidemiological studies. Eligibility criteria for enrolment in the registry are age $\geqslant 18$ years, severe AAT deficiency (PiZZ, PiZ Null and PiNull Null) and written informed consent. Clinical examination, blood samples (liver function) and lung function tests are performed at the patient's local hospital. Thereafter, the results are reported to the registry via a questionnaire which is answered by the attending physician every 2 years. Since 1999, all newly diagnosed subjects are also included in the Alpha One International Registry (AIR). ${ }^{15}$

The Pi phenotypes are verified by isoelectric focusing at the Department of Clinical Chemistry, University Hospital Malmö. ${ }^{16}$ All individuals were identified as phenotype PiZZ (few possible cases of PiZ Null, but none of PiNull Null). Phenotypes were available for all cases.

The indications for plasma protein analysis leading to PiZZ diagnosis were obtained from the questionnaire. The patients identified because of respiratory diseases or symptoms, including repeated respiratory tract infections, are referred to as "respiratory cases" (R cases). The patients identified because of liver diseases, other diseases and family/ population screening are referred to as "non-respiratory cases" (NR cases). The subgroup "other diseases" includes renal diseases, joint symptoms, repeated infections other than respiratory tract infection, high sedimentation rate, or other signs and symptoms for which plasma protein analysis has been performed as part of the clinical investigation. All symptoms and diagnoses were reported by the attending physician. 
Standard lung function tests were performed at the local hospitals. Forced expiratory volume in $1 \mathrm{~s}\left(\mathrm{FEV}_{1}\right)$ and forced vital capacity (FVC) are expressed as percentage of predicted values according to European reference tables. ${ }^{17}$ The $\mathrm{FEV}_{1} / \mathrm{FVC}$ ratio is expressed as a percentage. Results of the first spirometric measurement at the time of enrolment in the registry were used. Post-bronchodilator values were analysed.

Details of smoking habits were also obtained from the questionnaire. The patients investigated in this study were lifelong non-smokers. The patient's employment at the time of enrolment was obtained from the questionnaire and classified into various occupational categories according to the Standard Swedish Classification of Occupational Categories compiled by Statistics Sweden.

The Swedish AAT Registry is approved by the local research and ethics committee (Institutional Ethics Committee, Lund University, Sweden) and by the Swedish Data Inspection Board. Written informed consent was obtained from each subject prior to inclusion in the register.

\section{Causes of death}

The date of death was obtained from the Swedish Registry of Deaths. Vital status was available for all patients at the closing point of the study (1 September 2007). Information about liver transplantation and lung transplantation was obtained from the questionnaire and medical records. For each death, the medical records from the hospital at which the death occurred were reviewed. These records included medical charts from the terminal hospitalisation, last outpatient visits (if the patient died at home) and the death certificate. An autopsy protocol for patients who had undergone complete post-mortem examination was obtained from the Department of Pathology at which the autopsy was performed. All available records and autopsy protocols were analysed to determine the underlying and immediate causes of death. The underlying cause of death was defined as "the single disease or injury that influenced the events causing death". The immediate cause of death was defined as "the single final disease, injury or complication directly causing death".

\section{Statistical analysis}

The period of follow-up for the survival calculation was taken from the date of enrolment in the registry to the date of death, date of transplantation or to 1 September 2007 (closing date of study). The $\chi^{2}$ test was used to compare categorical variables and the Student $t$ test was used for group comparisons. Postbronchodilator spirometry data are presented as medians with

Table 1 Demographic data of study population

\begin{tabular}{lccc}
\hline & $\begin{array}{l}\text { All } \\
(\mathbf{N}=\mathbf{5 6 8})\end{array}$ & $\begin{array}{l}\text { R cases } \\
(\mathbf{N}=\mathbf{1 5 6})\end{array}$ & $\begin{array}{c}\text { NR cases } \\
(\mathbf{N}=\mathbf{4 1 2})\end{array}$ \\
\hline $\mathrm{M} / \mathrm{F}$ & $269 / 299$ & $77 / 79$ & $192 / 220$ \\
Deaths, n (\%) & $93(16)$ & $52(33)$ & $41(10)$ \\
Liver transplant, $\mathrm{n}$ & 3 & - & 3 \\
Lung transplant, $\mathrm{n}$ & 1 & 1 & - \\
Median (range) age at diagnosis (years) & $38(0-88)$ & $60(0.7-88)$ & $28(0-84)$ \\
Median (range) age at inclusion (years) & $43(18-89)$ & $63(18-89)$ & $33(18-87)$ \\
Median (range) years between diagnosis & $2(0.1-32)$ & $1(0.1-27)$ & $5(0.1-32)$ \\
and inclusion & & & \\
Median (range) age at death (years) & $76(24-95)$ & $76(30-95)$ & $75(24-90)$ \\
Median (range) follow-up time (years) & $11(0.1-16)$ & $7(0.1-16)$ & $12(0.4-16)$ \\
\hline
\end{tabular}

$\mathrm{R}$, respiratory; NR, non-respiratory. upper and lower quartile values $\left(\mathrm{P}_{25}-\mathrm{P}_{75}\right)$. To compare the death rates in the never-smoking $\mathrm{PiZZ}$ individuals with the general Swedish population, the specific standardised mortality rate (SMR) was calculated as the ratio of observed to expected deaths. The expected numbers of deaths were obtained using age-, sex- and date-specific death rates published in Sweden annually (Statistics Sweden). ${ }^{18}$ Confidence intervals for SMR were computed from the Poisson distribution. A $p$ value of $<0.05$ was considered significant.

\section{RESULTS}

\section{Study population}

Up to September 2007 a total of 1297 PiZZ individuals were included in the registry. Of these, 568 (44\%) were neversmokers and were included in the statistical analysis. One hundred and fifty-six subjects (27\%) were R cases and 412 were NR cases. Among the 412 NR cases, 56 individuals (14\%) had been identified because of liver disease, 169 (41\%) because of other disease and 187 (45\%) by family/population screening.

The demographic baseline data are shown in table 1; $47 \%$ of the study population were men, with $49 \%$ among the $\mathrm{R}$ cases and $47 \%$ among the NR cases. The R cases were older at the time of diagnosis and enrolment in the registry than the NR cases. The NR cases were followed up longer than the $\mathrm{R}$ cases.

\section{Lung function}

Post-bronchodilator spirometry data were available for $90 \%$ of the $\mathrm{R}$ cases and $93 \%$ of the NR cases. The results of the lung function tests stratified by age at inclusion in the registry are shown in table 2. The median $\mathrm{FEV}_{1}$ (\% predicted) was significantly higher in the NR cases than in the $R$ cases $(p<0.001)$. On average, study subjects aged $<50$ years had normal lung function in both groups while, in individuals aged $\geqslant 50$ years, lung function was significantly lower than in the younger age group $(p<0.001$, table 2$)$.

There was no significant difference in the spirometric values between men and women, the median $\left(\mathrm{P}_{25}-\mathrm{P}_{75}\right) \mathrm{FEV}_{1}(\%$ predicted) was 94 (77-105) and 93 (78-105), respectively. When the lung function tests for men and women were stratified by age at inclusion, there was no significant difference between men and women aged <50 years (median $\mathrm{FEV}_{1} 98$ (90-107)\% predicted and $100(91-108) \%$ predicted, respectively; $p=0.2)$. In contrast, women aged $\geqslant 50$ years had significantly higher $\mathrm{FEV}_{1}$ values than men in the same age subgroup, with a median $\mathrm{FEV}_{1}$ of $81(59-97) \%$ predicted and $67(47-93) \%$ predicted, respectively $(p<0.01)$.

\section{Respiratory diseases and symptoms at inclusion}

Of the $156 \mathrm{R}$ cases, 74 (47\%) were reported to have pulmonary emphysema at inclusion, 33 (21\%) had chronic bronchitis, 26 $(17 \%)$ asthma, $4(3 \%)$ pulmonary fibrosis, $6(4 \%)$ bronchiectasis and $13(8 \%)$ were reported to have other respiratory symptoms such as cough, phlegm or one or more attacks of pneumonia. Thirty-seven individuals in the $\mathrm{R}$ group were aged $<50$ years at inclusion. Their lung diseases and symptoms were pulmonary emphysema $(n=5)$, chronic bronchitis $(n=7)$, asthma $(n=17)$, bronchiectasis $(n=1)$ and other respiratory symptoms $(n=7)$. In 94 of the $412 \mathrm{NR}$ cases (23\%) a diagnosis of respiratory diseases or respiratory symptoms was reported at the time of inclusion. Of these $94 \mathrm{NR}$ cases, 23 (24\%) were reported to have pulmonary emphysema, 13 (14\%) chronic bronchitis, 42 (45\%) asthma, 2 (2\%) pulmonary fibrosis, $6(6 \%)$ bronchiectasis and in $8(9 \%)$ other respiratory symptoms were reported. Their median 
Table 2 Median $\left(\mathrm{P}_{25}-\mathrm{P}_{75}\right)^{*}$ post-bronchodilator $\mathrm{FEV}_{1}, \mathrm{FVC}$ and $\mathrm{FEV}_{1} / \mathrm{FVC}$ ratio in the respiratory $(\mathrm{R})$ and nonrespiratory (NR) cases stratified by age at inclusion

\begin{tabular}{|c|c|c|c|c|}
\hline & All & $\mathbf{R}$ cases & NR cases & p Value \\
\hline All age groups & $\mathrm{n}=524$ & $\mathrm{n}=141$ & $\mathrm{n}=383$ & \\
\hline $\mathrm{FEV}_{1}(\%$ predicted $)$ & $93(78-105)$ & $69(49-93)$ & 97 (87-107) & $<0.001$ \\
\hline FVC (\% predicted) & $92(80-100)$ & $86(68-97)$ & $93(84-101)$ & $<0.001$ \\
\hline $\mathrm{FEV}_{1} / \mathrm{FVC}(\%)$ & $80(68-87)$ & $56(40-76)$ & $83(77-89)$ & $<0.001$ \\
\hline Age at inclusion $<50$ years & $\mathrm{n}=320$ & $\mathrm{n}=37$ & $\mathrm{n}=283$ & \\
\hline $\mathrm{FEV}_{1}(\%$ predicted $)$ & $98(90-107)$ & 94 (85-109) & $99(90-107)$ & 0.2 \\
\hline FVC (\%predicted) & $94(87-102)$ & $92(83-102)$ & $95(88-102)$ & 0.5 \\
\hline $\mathrm{FEV}_{1} / \mathrm{FVC}(\%)$ & $85(80-89)$ & $83(77-90)$ & $85(80-89)$ & 0.2 \\
\hline Age at inclusion $>50$ years & $\mathrm{n}=204$ & $\mathrm{n}=104$ & $\mathrm{n}=100$ & \\
\hline $\mathrm{FEV}_{1}(\%$ predicted $)$ & 77 (54-95) & $59(43-80)$ & $90(75-105)$ & $<0.001$ \\
\hline FVC (\%predicted) & $83(69-96)$ & $79(64-96)$ & $85(73-96)$ & $<0.050$ \\
\hline $\mathrm{FEV}_{1} / \mathrm{FVC}(\%)$ & $63(50-77)$ & $51(38-65)$ & $76(62-82)$ & $<0.001$ \\
\hline
\end{tabular}

*Upper and lower quartile values.

$\mathrm{FEV}_{1}$, forced expiratory volume in $1 \mathrm{~s}$; FVC, forced vital capacity; R, respiratory; NR, non-respiratory.

age (range) at onset of the respiratory symptoms was $45(12-$ 76) years vs $52(4-82)$ years in the $R$ cases $(p<0.01)$.

\section{Occupation}

The patient's occupation was known in $87 \%(n=493)$ of the subjects (77\% in the R cases and $90 \%$ in the NR cases); $38 \%$ $(n=45)$ of the $R$ cases and $18 \%(n=68)$ of the NR cases had an occupation with a risk for exposure to airway irritants including agriculture, forestry, mining, trades and crafts, operation and assembly of machines and transport $(p=0.001)$. In the group with risk for exposure there were more men than women $(80 \%$ and $20 \%$, respectively).

\section{Survival}

During the follow-up period 93 individuals (16\%) died, 52 (33\%) of the $156 \mathrm{R}$ cases and $41(10 \%)$ of the $412 \mathrm{NR}$ cases. Among the NR cases, $12(21 \%)$ of the 56 individuals identified by liver disease, $26(15 \%)$ of the 169 individuals identified by other diseases and $3(2 \%)$ of the 187 individuals identified by screening died during the follow-up. The median age at death was 76 years for the $R$ cases and 75 years for the NR cases, with no significant difference. The SMR for the whole study population was 2.32 (95\% confidence interval (CI) 1.87 to 2.83). There was no significant difference in SMR between men and women, with values of 2.31 (95\% CI 1.66 to 3.12 ) and 2.32 (95\% CI 1.74 to 3.04), respectively. The SMR was 2.55 (95\% CI 1.91 to 2.83 ) for the R cases and 2.07 (95\% CI 1.49 to 2.81) for the NR cases. Further calculation of SMR for subgroups in the NR cases

Table 3 Underlying causes of death in 93 individuals who had never smoked

\begin{tabular}{|c|c|c|c|}
\hline & $\begin{array}{l}\text { All } \\
(n=93)\end{array}$ & $\begin{array}{l}R \text { cases } \\
(n=52)\end{array}$ & $\begin{array}{l}\text { NR cases } \\
(n=41)\end{array}$ \\
\hline & n (\%) & n (\%) & n (\%) \\
\hline Emphysema & $42(45)$ & $34(65)$ & $8(19)$ \\
\hline Liver diseases & $26(28)$ & $11(21)$ & $15(37)$ \\
\hline Other & $25(27)$ & $7(14)$ & $18(44)$ \\
\hline Malignancy & 13 & 4 & 9 \\
\hline Cardio/cerebrovascular diseases & 6 & 1 & 5 \\
\hline Gastrointestinal bleeding* & 2 & 1 & 1 \\
\hline Renal diseases & 2 & 1 & 1 \\
\hline Accident & 2 & - & 2 \\
\hline
\end{tabular}

*Without known liver disease.

$R$, respiratory, NR, non-respiratory. showed that SMR was 5.00 (95\% CI 2.58 to 8.73) for individuals identified by liver diseases, 1.98 (95\% CI 1.30 to 2.91) for individuals identified by other diseases and 0.70 (95\% CI 0.14 to 2.04) for individuals identified by family/population screening.

\section{Causes of death}

Medical records were available for all 93 decedents. Autopsies were performed in 18 cases (19\%) and autopsy protocols were available for all of these cases. The most common underlying causes of death were emphysema in $45 \%$ and liver disease in $28 \%$ (table 3). Malignancy accounted for $14 \%$ of the deaths. The underlying malignant diseases were pulmonary carcinoma $(n=3)$, urinary-genital carcinoma $(n=3)$, gastrointestinal malignancy $(n=4)$, melanoma $(n=1)$, lymphoma $(n=1)$ and pancreatic carcinoma $(n=1)$. Cardio/cerebrovascular diseases and renal diseases accounted for the remainder.

The immediate causes of death in the $R$ and NR cases are shown in table 4. Respiratory failure with or without pulmonary infection was the most common immediate cause of death ( $40 \%$ of cases). Hepatic complications due mostly to liver cirrhosis accounted for $24 \%$ of the immediate causes of death. The majority of the NR cases (53\%) died of causes other

Table 4 Immediate causes of death in 93 individuals who had never smoked

\begin{tabular}{|c|c|c|c|}
\hline & $\begin{array}{l}\text { All } \\
(n=93)\end{array}$ & $\begin{array}{l}R \text { cases } \\
(n=52)\end{array}$ & $\begin{array}{l}\text { NR cases } \\
(n=41)\end{array}$ \\
\hline & n (\%) & n (\%) & n (\%) \\
\hline Respiratory & $37(40)$ & $31(60)$ & $6(15)$ \\
\hline Respiratory failure & 25 & 21 & 4 \\
\hline Pneumonia & 12 & 10 & 2 \\
\hline Hepatic & $22(24)$ & $9(17)$ & $13(32)$ \\
\hline Liver failure & 6 & 3 & 3 \\
\hline Primary liver carcinoma & 11 & 5 & 6 \\
\hline Variceal bleeding & 4 & - & 4 \\
\hline Intra-abdominal infection & 1 & 1 & - \\
\hline Other & $34(36)$ & $12(23)$ & $22(53)$ \\
\hline Malignancy complication & 13 & 4 & 9 \\
\hline Cardio/cerebrovascular accident & 14 & 5 & 9 \\
\hline Intra-abdominal infection* & 1 & 1 & - \\
\hline Gastrointestinal bleeding* & 2 & 1 & 1 \\
\hline Suicide/accident & 2 & - & 2 \\
\hline Renal failure & 2 & 1 & 1 \\
\hline
\end{tabular}

*Without known liver disease.

$R$, respiratory; NR, non-respiratory. 
than respiratory and hepatic complications, such as a cardio/ cerebrovascular incident or malignancy.

\section{Liver disease among the deceased}

Twenty-nine patients who died (31\%) had liver disease. Of these, 12 cases had liver cirrhosis at enrolment in the register, 8 cases developed liver disease during the follow-up period ( 6 primary liver carcinoma diagnosed by biopsy and 2 liver cirrhosis diagnosed clinically), and the remaining 9 cases were diagnosed as having liver disease at autopsy ( 5 primary liver carcinoma and 4 liver cirrhosis). The median time between diagnosis and death for patients with primary liver carcinoma ranged from a few weeks to a few months.

The median age at death was 70 years (range 24-84) for the patients with liver disease. Liver diseases were the underlying cause of death in 26 of these 29 cases. In the remainding 3 cases the underlying causes of death were pulmonary carcinoma $(\mathrm{n}=1)$ and accident $(\mathrm{n}=2)$.

\section{DISCUSSION}

This study included a large number of PiZZ individuals who had never smoked and most of them had been identified by reasons other than respiratory symptoms. These individuals were followed-up prospectively from 1991 to 2007 using the Swedish AAT Registry. ${ }^{12}$ We found that never-smoking PiZZ individuals identified by respiratory symptoms, liver diseases and other diseases had a higher mortality risk than those identified by family/population screening. Thus, the screening group did not have an increased mortality risk compared with the normal Swedish population. This finding is in accordance with the findings in previous studies by Seersholm et $a l^{13} 14$ showing that the non-index cases did not have an excess mortality compared with the normal Danish population in spite of the fact that these studies included only a limited number of non-index cases.

Previously published studies of never-smoking PiZZ individuals are sparse and included mostly patients with respiratory symptoms at the time of diagnosis. Black et al reported a relatively good prognosis for PiZZ never-smokers who avoided known respiratory irritants. ${ }^{19}$ This study included only 22 individuals. The study by Seersholm et a ${ }^{14}$ showing that most PiZZ never-smokers had a normal life without respiratory symptoms included only 75 never-smokers.

In agreement with previously published studies, we found that emphysema is the underlying cause of death in most neversmokers (45\%). Seersholm et al reported that emphysema was the cause of death in 14 of 21 never-smokers who died. ${ }^{14}$ Stoller et $a l^{20}$ found that emphysema was the main underlying cause of death in 9 of 16 never-smokers.

An important finding in our study was the predominant role of cirrhosis-related mortality in elderly never-smokers. Cirrhosis and its complications were the main cause of death in 26 of 93 never-smokers and occurred in 29 of 93 patients. Malignant transformation was found in $38 \%$ of cases with cirrhosis. These findings agree with the most recent data which suggest that cirrhosis and carcinoma of the liver affect $30-40 \%$ of PiZZ individuals over the age of 50 years and are a significant cause of death in never-smokers. ${ }^{9-11}$ Eriksson reported that cirrhosis was the main cause of death in 12 of $41 \mathrm{PiZZ}$ individuals who died in Malmö, Sweden; cirrhosis found at the post-mortem examination was more common among elderly never-smokers (71\%). Malignant transformation was seen in 5 of 14 cases of cirrhosis. ${ }^{11}$ Our findings agree closely with those of Eriksson, suggesting that the risk of cirrhosis is increased in elderly PiZZ never-smokers and that cirrhosis should be considered as a premalignant condition.

Previous studies have consistently shown that lung function is well preserved in early adult life in non-smoking PiZZ individuals. ${ }^{12} 2122$ In our study, most never-smokers seemed to have normal lung function until 50 years of age. The deterioration in lung function seems to begin after this age in the $\mathrm{R}$ cases, while NR cases maintain their normal lung function after the age of 50 years. Our previous study showed similar results, but the number of NR cases over the age of 50 was small and none of them was older than 61 years. ${ }^{12}$ In the present study, $30 \%$ of the NR cases were aged $>50$ years and $17 \%$ were $>60$ years of age.

Twenty-three percent of the NR cases had respiratory disease at the time of enrolment. Most of these patients were identified by neonatal/population screening. Among them, there was a high prevalence of asthma (45\%) which had been diagnosed by the attending physician during childhood or early adulthood. These findings explain the earlier onset of respiratory symptoms (45 years of age) in this subgroup of NR cases compared with the $\mathrm{R}$ cases (52 years of age).

It is still unclear why some PiZZ individuals develop severe lung disease and others remain asymptomatic in the absence of the important risk factor for the development of emphysema (ie, active smoking). We found that the group of $\mathrm{R}$ cases included significantly more individuals with high-risk occupations than the group of NR cases. There was also a male predominance among the group with high-risk occupations. This finding may explain the impairment of lung function in $\mathrm{R}$ cases and in men aged $>50$ years. However, only current employment status was reported and no details of duration of exposure to airway irritants were available.

The first limitation of our study is the fact that it is a register-based study and the subjects included in the Swedish AAT Registry do not represent a random sample of adult PiZZ individuals living in Sweden. However, the proportion of the individuals identified for reasons other than respiratory symptoms is higher than in other registries. The second limitation is that smoking habits were self-reported and no objective tests such as the cotinine level in plasma or urine were analysed. The third limitation is that we compared the SMR for the study population with SMR for the general Swedish population because no data are available on mortality in the subgroups based on smoking habits in the Swedish general population.

We conclude that never-smoking PiZZ individuals seem to have a higher mortality risk than the Swedish general population except for individuals identified by family/ population screening who seem to have the same mortality rate as the general population. The risk of liver cirrhosis and liver carcinoma is high in elderly never-smoking PiZZ individuals.

Acknowledgements: The authors thank Maria Walles for secretarial assistance, Dr Tomas Sveger for support and advice and all the Swedish physicians who report data to the Swedish AAT Deficiency Registry.

Funding: This work was supported by grants from the Swedish National Heart-Lung Foundation.

Competing interests: None.

Ethics approval: The Swedish AAT Registry is approved by the local research and ethics committee (Institutional Ethics Committee, Lund University, Sweden) and by the Swedish Data Inspection Board. Written informed consent was obtained from each subject prior to inclusion in the register. 


\section{REFERENCES}

1. American Thoracic Society/European Respiratory Society. Statement: standards for the diagnosis and management of individuals with alpha-1 antitrypsin deficiency. Am J Respir Crit Care Med 2003;168:818-900.

2. Brantly M, Nukiwa T, Crystal RG. Molecular basis of alpha-1-antitrypsin deficiency. Am J Med 1988;84:13-31.

3. Cox DW, Billingsley GD, Mansfield T. DNA restriction-site polymorphisms associated with the alpha 1-antitrypsin gene. Am J Hum Genet 1987:41:891-906.

4. Eriksson S. Studies in alpha 1-antitrypsin deficiency. Acta Med Scand Supp/ 1965:432:1-85.

5. Tobin MJ, Cook PJ, Hutchison DC. Alpha 1 antitrypsin deficiency: the clinical and physiological features of pulmonary emphysema in subjects homozygous for Pi type Z. A survey by the British Thoracic Association. Br J Dis Chest 1983;77:14-27.

6. Larsson C. Natural history and life expectancy in severe alpha-1-antitrypsin deficiency, Pi Z. Acta Med Scand 1978:204:345-51.

7. Sharp H. Alpha-one antitrypsin deficiency. Hosp Pract 1971;6:83.

8. Sveger T. Liver disease in alpha1-antitrypsin deficiency detected by screening of 200,000 infants. N Engl J Med 1976;294:1316-21.

9. Eriksson S, Carlson J, Velez R. Risk of cirrhosis and primary liver cancer in alpha 1antitrypsin deficiency. N Engl J Med 1986;314:736-9.

10. Elzouki AN, Eriksson S. Risk of hepatobiliary disease in adults with severe alpha 1antitrypsin deficiency (PiZZ): is chronic viral hepatitis B or $\mathrm{C}$ an additional risk factor for cirrhosis and hepatocellular carcinoma? Eur J Gastroenterol Hepatol 1996;8:989-94.

11. Eriksson S. Alpha-1 antitrypsin deficiency: natural course and therapeutic strategies. In: Boyer J, Blum HE, Maier KP, et al., eds Cirrhosis and its development. Falk Symposium 115. Dordrecht, The Netherlands: Kluwer Academic, 2000:305-13.
12. Piitulainen E, Tornling G, Eriksson S. Effect of age and occupational exposure to airway irritants on lung function in non-smoking individuals with alpha 1-antitrypsin deficiency (PiZZ). Thorax 1997;52:244-8.

13. Seersholm N, Kok-Jensen A, Dirksen A. Survival of patients with severe alpha 1 antitrypsin deficiency with special reference to non-index cases. Thorax 1994; 49:695-8.

14. Seersholm N, Kok-Jensen A. Clinical features and prognosis of life time nonsmokers with severe alpha 1-antitrypsin deficiency. Thorax 1998;53:265-8.

15. Stockley RA, Luisetti M, Miravitlles M, et al. Alpha-1-antitrypsin deficiency International Registry group. Ongoing research in Europe: Alpha-1-antitrypsin deficiency International Registry (AIR) objectives and development. Eur Respir J 2007;29:582-6.

16. Jeppsson J0, Franzen B. Typing of genetic variants of alpha 1-antitrypsin by electrofocusing. Clin Chem 1982;28:219-25.

17. Quanjer P. Standardized lung function testing. Official statement of the European Respiratory Society. Eur Respir J Supp/ 1993;16:1-100.

18. Statistics Sweden (Bureau of Statistics in Stockholm). 1991-2006. www.ssd. scb.se.

19. Black LF, Kueppers F. Alpha-1-Antitrypsin deficiency in nonsmokers. Am Rev Respir Dis 1978;117:421-8.

20. Stoller JK, Tomashefski J Jr, Crystal RG, et al. Mortality in individuals with severe deficiency of alpha1-antitrypsin: findings from the National Heart, Lung, and Blood Institute Registry. Chest 2005:127:1196-204.

21. Bernspang $\mathbf{E}$, Sveger T, Piitulainen E. Respiratory symptoms and lung function in 30 year-old individuals with alpha-1-antitrypsin deficiency. Respir Med 2007;101:1971-6.

22. Silverman EK, Pierce JA, Province MA, et al. Variability of pulmonary function in alpha-1-antitrypsin deficiency: clinical correlates. Ann Intern Med 1989;111:982-91.

\section{Lung alert}

\section{A new theory for the pathogenesis of chronic lung disease?}

Chronic inflammatory disease is generally thought to be the result of an altered adaptive immune response. This paper describes a novel innate immune pathway driven by persistent viral infection.

A mouse model of chronic inflammatory lung disease was generated by a viral infection which produced mucous cell metaplasia and airway hyper-responsiveness. Maximal airway disease activity coincided with a peak of CD1d-dependent $\mathrm{T}$ cell receptor-invariant natural killer $\mathrm{T}$ (NKT) cell recruitment of macrophages as well as peak interleukin (IL)13 levels in the lungs. This cellular interaction was independent of CD4+ T cells which were previously thought to be the focus of a response to allergens in the lungs.

It has long been suggested that common respiratory viruses are central to the development of chronic airway inflammation in asthma, particularly in children. However, it is not clear how this could occur many years after the apparent clearance of the infection. Interestingly, this report shows that the virus initiating the airway damage remains detectable in the lung in trace amounts, and further studies will be needed to establish the relevance of persistent infection in those with chronic obstructive pulmonary disease.

The discovery of this new immune axis suggests that the recruitment and persistent activation of IL13 producing macrophages by NKT cells in human lung tissue may be involved in the development of chronic lung disease.

- Kim EY, Battaile JT, Patel AC, et al. Persistent activation of an innate immune response translates respiratory viral infection into chronic lung disease. Nat Med 2008;14:633-9

\section{Hill}

Correspondence to: Dr M Hill, F2, Kings' College Hospital, London, UK; martinhill@doctors.net.uk 